\title{
Factores asociados al bajo peso al nacer en un hospital universitario del departamento de Nariño
}

\author{
Factors associated with low birth weight at a university hospital from the department of Nariño (Colombia) \\ Yusley Katerine Pabón-Salazar1* orcid.org/0000-0002-6067-1098 \\ Jenny Patricia Eraso-Revelo² orcid.org/0000-0003-3623-8283 \\ Gustavo Bergonzoli-Pelaez ${ }^{3}$ orcid.org/0000-0001-9728-5329
}

Andry Yasmid Mera-Mamián ${ }^{4}$ orcid.org/0000-0002-2356-3370

1. Universidad del Cauca. Popayán, Colombia

2. Hospital Universitario Departamental de Nariño. Pasto, Colombia

3. Hospital Tomás Uribe; Fundación para la Producción y Gestión del Conocimiento (PROGESCO). Cali, Colombia

4. Universidad CES. Medellín, Colombia

Pabón-Salazar YK, Eraso-Revelo JP, Bergonzoli-Pelaez G, Mera-Mamián AY. Factores asociados al bajo peso al nacer en un hospital universitario del departamento de Nariño. Univ. Salud. 2021;23(3):179-188. DOI: https://doi.org/10.22267/rus.212303.231

\section{Resumen}

Introducción: El Bajo Peso al Nacer (BPN) tiene múltiples causas y continúa siendo un problema que afecta diferentes regiones de Colombia. Objetivo: Determinar los factores asociados con BPN en el Hospital Universitario Departamental de Nariño. Materiales y métodos: Estudio de casos y controles que incluyó madres y recién nacidos, cuyo parto fue atendido entre noviembre de 2014 y junio de 2015. Resultados: El riesgo de BPN fue mayor en mujeres con edad entre 10-19 años $(O R=7,79, I C 95 \%=2,61-23,23)$, con antecedente de infección vaginal durante el embarazo $(O R=4, I C 95 \%=1,26-12,66)$, nivel educativo primaria incompleta $(O R=10,93$, IC95\%=1,51-79,13) o primaria completa $(O R=2,94, I C 95 \%=1,06-8,13)$, afiliadas al régimen de salud subsidiado $(O R=4,96$, $I C 95 \%=1,71-14,41)$. El riesgo de tener un recién nacido con BPN se redujo un $10 \%(O R=0,90$, IC $95 \%=0,84-0,96)$ por cada incremento en una unidad de Índice de Masa Corporal y un 76\% (OR=0,23, IC95\%=0,16-0,34) por cada incremento en una semana de gestación. Conclusiones: La mayoría de factores de riesgo identificados pueden ser prevenidos o intervenidos precozmente desde un enfoque de salud pública.

Palabras clave: Recién nacido de bajo peso; embarazo; atención prenatal; factores de riesgo; modelos logísticos. (Fuente: DeCS, Bireme).

\begin{abstract}
Introduction: Low Birth Weight (LBW) has multiple causes and continues to be a problem that affects different regions of Colombia. Objective: To determine factors associated with LBW in the Departmental University Hospital of Nariño (Colombia). Materials and methods: A case-control study that included newborns and mothers whose delivery took place between November 2014 and June 2015. Results: Risk of LBW was higher in: women aged between 10-19 years $(O R=7.79,95 \% C I=2.61-23.23)$; with history of vaginal infection during pregnancy $(O R=4,95 \% C I=1.26-12.66)$; and those affiliated with the subsidized health regimen $(O R=4.96$, $95 \% C I=1.71-14.41)$. The risk of having a newborn with LBW was reduced by $10 \%(O R=0.90,95 \% C I=0.84-0.96)$ for each one unit increase in Body Mass index, and by 76\% (OR=0.23, 95\% CI=0.16-0.34) for each increase in one week of gestation. Conclusions: Most of the identified risk factors can be prevented or intervened with through an early public health approach.
\end{abstract}

Keywords: Low birth weight infant; pregnancy; prenatal care; risk factors; logistic models. (Source: DeCS, Bireme).

\footnotetext{
*Autor de correspondencia

Yusley Katerine Pabon Salazar

e-mail: yusley@unicauca.edu.co
} 


\section{Introducción}

Según la Organización Mundial de la Salud (OMS) el recién nacido de bajo peso (BPN) es aquel neonato con peso menor a 2500 gramos (gr) ${ }^{(1)}$. En países como Brasil se ha reportado que los bebés que nacen con un peso entre 1500 gr y $2499 \mathrm{gr}$, enfrentan un riesgo de muerte neonatal aproximadamente 5 veces mayor $(\mathrm{IR}=5,76, \mathrm{IC} 95 \%=4,99-6,65)$ que aquellos que pesan entre 3000gr y 3999gr, y que el riesgo es aproximadamente 40 veces mayor (IR=41,1, IC95\%= $35,31-47,83)$ en recién nacidos que pesan entre 1000 y 1499gr (muy bajo peso al nacer) ${ }^{(2)}$.

Las causas son múltiples y están relacionadas con características maternas, demográficas y socioculturales. Entre los factores reportados asociados al BPN están: la edad materna $\leq 19$ años y $\geq 35$ años, la multiparidad, el bajo nivel educativo, el estado civil soltera, la asistencia prenatal de mala calidad y los que interfieren con la nutrición del feto y ganancia de peso ${ }^{(3-5)}$.

El BPN puede ocurrir por parto prematuro y/o retardo en el crecimiento intrauterino, el primero puede presentarse por infecciones del tracto genital, de las vías urinarias, anemia, preeclampsia y ruptura prematura de membranas, que generan incapacidad del útero para retener el feto(6); por su parte, el retardo de crecimiento intrauterino puede estar relacionado con factores asociados con la salud del útero y de la madre, entre ellos el estado nutricional materno y los determinantes sociales y ambientales de la salud(7). Según lo reportado en los lineamientos del Programa Madre Canguro del año 2017, en Colombia, el $12 \%$ de los nacimientos son prematuros, lo que significa alrededor de 100.000 niños al año con esta condición(8).

En Colombia, la prevalencia de BPN varió entre $8,76 \%, 8,80 \%, 8,81 \%$ y $9,13 \%$, en los años 2008, 2015, 2012 y 2018, respectivamente; estas cifras varían entre las regiones del país, territorios como Bogotá, D,C, Cundinamarca y Boyacá, presentaron prevalencias de BPN superiores al promedio nacional, en el 2018; para este mismo año el departamento de Nariño reportó 9,63\%, cifra que representa un aumento respecto a años anteriores $(9,41 \% \text { en } 2016 \text { y } 9,18 \% \text { en } 2017)^{(9)}$.

El Hospital Universitario Departamental de Nariño (HUDN), institución de referencia del departamento y de otras regiones, presentó durante el periodo 20152018, una prevalencia de BPN que varió entre $17 \%$, $17 \%, 18 \%$ y $21 \%$, respectivamente. En el año 2019 se presentaron 2053 nacimientos, de los cuales 376 (18\%) fueron clasificados como BPN.

Debido a que existe una amplia variabilidad en la prevalencia del BPN dentro de un mismo país, así como de los factores asociados, dependiendo de la región donde se presenta el evento, se considera importante para efectos de salud pública, identificar los factores responsables de la ocurrencia del BPN en una región determinada; este conocimiento contribuiría a la actualización de las políticas y programas aplicados para el cuidado del binomio madre-hijo.

Por lo anterior, se desarrolló una investigación que tuvo por objeto determinar los factores asociados al BPN en el HUDN.

\section{Materiales y métodos}

Se realizó un estudio de casos y controles con casos incidentes. El marco muestral correspondió a las madres y recién nacidos cuyo parto fue atendido en el HUDN, entre noviembre del año 2014 y junio del año 2015. Los criterios de inclusión fueron: madre residente del departamento de Nariño con producto único nacido vivo, independientemente de la edad gestacional. Se excluyeron recién nacidos con malformaciones congénitas como trisomía 13, 18 ó 21 y óbito fetal. Se contó con aval institucional para la revisión de historias clínicas.

Para el cálculo del tamaño de la muestra se usó la fórmula propuesta por Schlesselman ${ }^{(10)}$ y teniendo en cuenta una relación de caso: control de 1:1 se estudiaron 117 casos y 116 controles. La selección de los controles se hizo aleatoriamente. Al calcular el tamaño de muestra del estudio, se tuvo en cuenta un poder del $80 \%$, previendo que se presentaran problemas con el poder para detectar algún efecto específico en factores o variables, se aplicó la recomendación de Rothman(11) de cambiar el nivel alfa de 0,05 a 0,10 , lo cual incrementa el poder del estudio, por ello, los intervalos de confianza se reportaron al nivel del 90\%.

Se definió como caso incidente a todo recién nacido vivo cuya primera medición del peso al nacer fuera inferior a $2500 \mathrm{gr}$, y como control recién nacido vivo 
con peso igual o superior a $2500 \mathrm{gr}$, y nacido en la misma fecha del caso.

Se establecieron estrategias para el control de los sesgos, como selección de casos y de controles en el HUDN, el cual atiende un alto porcentaje de los partos, independientemente de la complejidad, por tratarse del hospital público de referencia en el departamento de Nariño. También se realizó estandarización de los formularios de recolección de información, las balanzas utilizadas en el área de partos fueron previamente calibradas. El personal encargado de recolectar los datos fue previamente entrenado y desconocían la condición de caso y control de los sujetos en estudio.

Se diseñó un formulario para la caracterización sociodemográfica y recolección de información relacionada con características del control prenatal, el cual se diligenció con información suministrada por las madres en el servicio de ginecobstetricia después del parto. Los datos clínicos y de laboratorio fueron tomados directamente de la historia clínica. Previo a su aplicación el instrumento fue sometido a una prueba piloto con tres encuestas de casos y tres controles por parte de los investigadores y los colaboradores en recolección de datos, donde se evaluó la redacción de las preguntas y su comprensión por parte de las entrevistadas. Se completó el formato con datos faltantes como fecha de nacimiento de la madre, identificación completa, número de historia clínica, se añadió la opción de no dato o no contesta en diferentes variables. Una vez incorporados los ajustes, se repitió la prueba piloto hasta contar con un instrumento final. Posteriormente se revisó la presencia de errores de digitación usando muestreo aleatorio simple, y se llevó a cabo un análisis exploratorio de datos.

\section{Análisis estadístico}

El análisis univariado de variables cualitativas se realizó mediante a través de frecuencia y porcentaje y las cuantitativas mediante el uso de las medidas de tendencia central y dispersión, realizando evaluación de los supuestos de normalidad, para definir el uso de estadística paramétrica o no paramétrica.

El análisis bivariado permitió identificar las variables que fueron incluidas en la fase analítica multivariada, en la fase bivariada se utilizó el test de independencia estadística, $T$ de Student para variables medidas en escala cuantitativas y el $C h i^{2}$ para las cualitativas; se tuvo en consideración un nivel de significancia del $10 \%$ y nivel de confianza 90\%, también se incluyó en el modelo logístico aquellas variables con significancia clínica, aunque no arrojaran significancia estadística. A las variables continuas se les realizó la prueba del logaritmo para definir como entrarían en el modelo logístico y si era necesario agruparlos en intervalos. En consecuencia, la edad de las madres en el momento del embarazo y la ganancia de peso durante la gestación, entraron en forma de intervalos, porque el resultado de la prueba de logaritmo fue $p<0,10$.

Se estimaron cuatro modelos logísticos agrupando las variables en tres determinantes en salud establecidos por Mark Lalonde(12): biológicos, estilos de vida y organización sanitaria y un último modelo en el que se incluyeron las variables que resultaron estadísticamente significativas en los tres primeros modelos; aquellas variables que posterior al ajuste continuaron siendo estadísticamente significativas se presentan como modelo final. Se calculó el Odds Ratio (OR) y su respectivo intervalo de confianza al 90\%. El análisis estadístico se realizó con el paquete IBM SPSS®, versión 20.

\section{Consideraciones éticas}

Al tratarse de una investigación con riesgo mínimo y teniendo en cuenta lo que establece la resolución 008430 de 1993 en su artículo 16, parágrafo primero "En el caso de investigaciones con riesgo mínimo, el Comité de Ética en Investigación de la institución investigadora, por razones justificadas, podrá autorizar que el Consentimiento Informado se obtenga sin formularse por escrito y tratándose de investigaciones sin riesgo, podrá dispensar al investigador de la obtención del mismo", no se tomó consentimiento informado, dado que la entrevista con las participantes solo pretendía completar algún dato faltante(13).

Esta investigación fue clasificada como de riesgo mínimo, según la resolución 008430 de 1993 del Ministerio de Salud de Colombia y contó con el aval del Comité de evaluación Ética y Científica en seres humanos de la Universidad Libre de Colombia, sede Cali, bajo Acta No, 010 del 10 de noviembre de 2014. 


\section{Resultados}

Características basales de los casos y los controles. Un total de 233 recién nacidos participaron en el estudio, 117 casos y 116 controles. Se encontró que casos y controles fueron similares respecto a las variables etnia y estado civil, siendo en su mayoría solteras y mestizas. El mayor porcentaje de la población ( $87 \%$ de casos y $81 \%$ de controles) pertenecía al estrato I, lo que para Colombia representa poblaciones que obtienen menor puntaje relacionado con características de las viviendas y su entorno urbano o rural, en general tanto estrato I, II y III albergan a los usuarios con menores recursos, los cuales son beneficiarios de subsidios en los servicios públicos domiciliarios (Tabla 1).

Tabla 1. Características sociodemográficas de la muestra, Nariño, 2015

\begin{tabular}{|c|c|c|c|c|c|c|c|}
\hline \multirow{2}{*}{ Variable } & \multicolumn{2}{|c|}{ Caso $(n=117)$} & \multicolumn{2}{|c|}{ Control $(n=116)$} & \multirow{2}{*}{$\begin{array}{c}\text { Estadístico } \\
\text { Chi }^{2}\end{array}$} & \multirow{2}{*}{ Gl } & \multirow{2}{*}{ Valor-p } \\
\hline & $\mathrm{n}$ & $\%$ & $\mathbf{n}$ & $\%$ & & & \\
\hline \multicolumn{8}{|l|}{ Estrato económico } \\
\hline Estratos II y III & 15 & 13 & 22 & 19 & 1,6 & 1 & 0,10 \\
\hline Estrato I & 102 & 87 & 94 & 81 & & & \\
\hline \multicolumn{8}{|l|}{ Etnia } \\
\hline Mestiza & 100 & 85 & 106 & 91 & 1,96 & 1 & 0,15 \\
\hline Afrocolombiana & 17 & 15 & 10 & 9 & & & \\
\hline \multicolumn{8}{|l|}{ Estado civil } \\
\hline Soltera & 42 & 36 & 41 & 35 & 1,08 & 2 & 0,58 \\
\hline Unión libre & 53 & 45 & 47 & 41 & & & \\
\hline Casada & 22 & 19 & 28 & 24 & & & \\
\hline
\end{tabular}

$\mathrm{Al}$ analizar la relación entre el BPN y cada una de las variables biológicas de interés, se encontró relación estadísticamente significativa con las variables: antecedentes personales de hipertensión, antecedente de BPN, ruptura prematura de membranas, vaginitis al inicio y al final de embarazo, anemia al inicio $y$ al final del embarazo, hospitalización en el embarazo, edad de la madre, ganancia de peso, periodo intergenésico, edad gestacional y alto riesgo obstétrico.

En cuanto a la edad gestacional el $64 \%$ fueron prematuros menores de 37 semanas y el 36\% a término: mayor o igual a 37 semanas. Por su parte el riesgo obstétrico se definió teniendo en cuenta los criterios del Centro Latinoamericanos de Perinatología (CLAP), el cual considera aspectos como antecedentes médicos y evolución del embarazo.

No se encontró relación estadísticamente significativa con las variables antecedente familiar y personal de diabetes, preeclampsia y antecedente familiar de hipertensión, diabetes e hipertensión gestacional, serología reactiva al inicio y al final de embarazo, infección de vías urinarias al inicio y al final del embarazo, gravidez, abortos, índice de masa corporal (IMC). La variable IMC se incluyó en el análisis multivariado por considerar su importancia clínica, aunque no fue estadísticamente significativa en el análisis bivariado. De acuerdo con el IMC al inicio del embarazo el 8,5\% de los casos y $3 \%$ de los controles clasificaron con peso bajo, el 63,2\% y $64 \%$ respectivamente peso normal, el $30 \%$ y $20,5 \%$ en sobrepeso y el $7,7 \%$ y $3 \%$ en obesidad grado I. No se encontraron madres con obesidad grado II ni obesidad mórbida.

Las variables del constructo teórico "estilos de vida", que resultaron estadísticamente significativas en el análisis bivariado fueron: el embarazo planeado, la procedencia, la ocupación, nivel educativo, la afiliación en salud y si fue remitida o no (Tabla 2). No resultaron estadísticamente significativas ser fumadora activa, fumadora pasiva, consumo de sustancias psicoactivas y alcohol.

A nivel de los servicios de salud, solo se consideró la variable número de controles prenatales, la cual fue estadísticamente significativa (Tabla 2). No resultó estadísticamente significativa la variable semana de inicio del control prenatal. 
Tabla 2. Análisis bivariado con variables biológicas, hábitos de vida y servicios de salud

\begin{tabular}{|c|c|c|c|c|c|c|c|c|}
\hline \multirow{2}{*}{$\begin{array}{c}\text { Variable } \\
\text { (n casos: } n \text { controles) }\end{array}$} & & \multicolumn{2}{|l|}{ Caso } & \multicolumn{2}{|c|}{ Control } & \multirow{2}{*}{$\begin{array}{l}\text { Valor del } \\
\text { estadístico }\end{array}$} & \multirow{2}{*}{$\begin{array}{c}\text { Grados } \\
\text { libertad }\end{array}$} & \multirow{2}{*}{ Valor-p } \\
\hline & & $\mathbf{n}$ & $\%$ & $\mathrm{n}$ & $\%$ & & & \\
\hline \multirow{2}{*}{$\begin{array}{l}\text { Antecedentes personales HTA } \\
(117: 116)\end{array}$} & $\mathrm{Si}$ & 6 & 5 & 0 & 0 & & 1 & $0,03 f$ \\
\hline & No & 111 & 95 & 116 & 100 & & & \\
\hline \multirow{2}{*}{$\begin{array}{l}\text { Antecedente bajo peso al } \\
\text { nacer }(32: 24)\end{array}$} & $\mathrm{Si}$ & 18 & 56 & 2 & 8 & 13,7 & 1 & $0,000 f$ \\
\hline & No & 14 & 44 & 22 & 92 & & & \\
\hline \multirow{2}{*}{$\begin{array}{l}\text { Ruptura prematura } \quad d d \\
\text { membranas }(117: 116)\end{array}$} & $\mathrm{Si}$ & 32 & 27 & 11 & 9 & 12,3 & 1 & $<0,0001^{x 2}$ \\
\hline & No & 85 & 73 & 105 & 91 & & & \\
\hline \multirow{2}{*}{$\begin{array}{l}\text { Vaginitis inicio embarazo } \\
(96: 93)\end{array}$} & $\mathrm{Si}$ & 39 & 41 & 25 & 27 & 3,9 & 1 & $0,04 \times 2$ \\
\hline & No & 57 & 59 & 68 & 73 & & & \\
\hline \multirow{2}{*}{$\begin{array}{l}\text { Vaginitis final embarazo } \\
\text { (103:101) }\end{array}$} & $\mathrm{Si}$ & 20 & 19 & 9 & 9 & 4,6 & 1 & $0,03 \times 2$ \\
\hline & No & 83 & 81 & 92 & 91 & & & \\
\hline \multirow{2}{*}{$\begin{array}{l}\text { Anemia inicio embarazo } \\
(58: 70)\end{array}$} & $\mathrm{Si}$ & 13 & 22 & 5 & 7 & 6,1 & 1 & $0,01 \times 2$ \\
\hline & No & 45 & 78 & 65 & 93 & & & \\
\hline \multirow{2}{*}{$\begin{array}{l}\text { Anemia final embarazo } \\
(117: 116)\end{array}$} & $\mathrm{Si}$ & 34 & 29 & 22 & 19 & 3,2 & 1 & $0,07 \times 2$ \\
\hline & No & 83 & 71 & 94 & 81 & & & \\
\hline \multirow{2}{*}{$\begin{array}{l}\text { Hospitalización embarazo } \\
(115: 115)\end{array}$} & $\mathrm{Si}$ & 64 & 56 & 37 & 32 & 12,8 & 1 & $<0,0001 \times 2$ \\
\hline & No & 51 & 44 & 78 & 68 & & & \\
\hline \multirow{2}{*}{$\begin{array}{l}\text { Riesgo obstétrico } \\
(117: 116)\end{array}$} & Alto & 81 & 69 & 47 & 40 & 19,39 & 1 & $<0,0001^{x 2}$ \\
\hline & Bajo & 36 & 31 & 69 & 59 & & & \\
\hline \multirow{3}{*}{$\begin{array}{l}\text { Edad madre } \\
(117: 116)\end{array}$} & 10 - 19 años & 30 & 25,6 & 16 & 13,8 & 5,198 & 2 & $0,074 \times 2$ \\
\hline & $20-29$ años & 58 & 49,6 & 68 & 58,6 & & & \\
\hline & $\geq 30$ años & 29 & 24,8 & 32 & 27,6 & & & \\
\hline \multirow{4}{*}{$\begin{array}{lllll}\text { Ganancia de peso en el } \\
\text { embarazo } & & & \\
(117: 115) & & & \end{array}$} & $<7 \mathrm{~kg}$ & 47 & 40,2 & 28 & 24,3 & 8,42 & 3 & $0,038^{x 2}$ \\
\hline & $7-10 \mathrm{~kg}$ & 24 & 20,5 & 39 & 33,9 & & & \\
\hline & $11-15 \mathrm{~kg}$ & 33 & 28,2 & 34 & 29,6 & & & \\
\hline & $\geq 16$ & 13 & 11,1 & 14 & 12,2 & & & \\
\hline \multicolumn{2}{|l|}{$\begin{array}{l}\text { Período intergenésico } \\
(68: 55)\end{array}$} & $\begin{array}{r}65,5 \\
(28,8-104,3)\end{array}$ & & $\begin{array}{r}96 \\
(50-126)\end{array}$ & & 1241 & & $0,006^{U}$ \\
\hline \multicolumn{2}{|l|}{$\begin{array}{l}\text { Edad gestacional } \\
(117: 116)\end{array}$} & $\begin{array}{r}35,6 \\
(33-37,4)\end{array}$ & & $\begin{array}{r}39 \\
(38-39,5)\end{array}$ & & 1,684 & & $<0,0001^{U}$ \\
\hline \multicolumn{9}{|l|}{ Hábitos de vida } \\
\hline \multirow{2}{*}{$\begin{array}{l}\text { Procedencia } \\
(117: 116)\end{array}$} & Pasto & 60 & 51 & 72 & 62 & 2,7 & 1 & $0,096^{\times 2}$ \\
\hline & $\begin{array}{r}\text { Otros } \\
\text { municipios }\end{array}$ & 57 & 49 & 44 & 38 & & & \\
\hline Ocupación & Ama de casa & 83 & 73 & 69 & 0 & 3,2 & 1 & $0,7 \times 2$ \\
\hline$(114: 112)$ & Otra ocupación & 31 & 27 & 43 & 38 & & & \\
\hline Nivel Educativo & P. incompleta & 9 & 8 & 3 & 2,6 & 15 & 4 & $0,005 \times 2$ \\
\hline$(115: 116)$ & P. completa & 26 & 23 & 17 & 27 & & & \\
\hline & S. incompleta & 38 & 33 & 25 & 21,6 & & & \\
\hline & S. completa & 27 & 23 & 46 & 37,7 & & & \\
\hline & Profesionales & 15 & 13 & 25 & 21,6 & & & \\
\hline Afiliación a salud & Contributivo & 19 & 17 & 38 & 34 & 7,83 & 1 & $0,005 \times 2$ \\
\hline$(110: 113)$ & Subsidiado & 91 & 83 & 75 & 66 & & & \\
\hline Remitida & $\mathrm{Si}$ & 62 & 53 & 29 & 25 & 19,7 & 1 & $<0,0001^{x 2}$ \\
\hline$(117: 116)$ & No & 55 & 47 & 87 & 75 & & & \\
\hline Embarazo planeado & No & 95 & 81 & 82 & 71 & 3,5 & 1 & $0,06 \times 2$ \\
\hline$(117: 116)$ & $\mathrm{Si}$ & 22 & 19 & 34 & 29 & & & \\
\hline Servicios de salud & & & & & & & & \\
\hline $\begin{array}{l}\text { Número controles prenatales } \\
(109: 116)\end{array}$ & & & $5(3-6)$ & & $(5-8)$ & 4641 & & $<0,0001^{U}$ \\
\hline
\end{tabular}




\section{Modelo biología humana (modelo 1)}

Después de correr la regresión logística binaria para el modelo 1, las variables con significancia estadística fueron: infección vaginal al final del embarazo, edad joven (10 - 19 años), IMC y edad gestacional a término. Se identificó que el riesgo de tener un recién nacido de BPN fue 2,95 veces en las mujeres que presentaban infección vaginal en la gestación comparado con aquellas que no; 4,24 veces en las mujeres que se embarazan en edades entre 10 y 19 años, comparados con las mujeres que se embarazan después de los 19 años. Por cada unidad de aumento en el IMC el riesgo de tener un recién nacido de bajo peso, presentó una reducción promedio de $7 \%$ y por cada semana de aumento en la edad gestacional, el riesgo de tener un recién nacido de bajo peso presentó una reducción global del 67\%.
El primer modelo clasificó correctamente al 84,8\% (173/233) de los individuos; 79,6\% (82/117) de los casos (Sensibilidad) y 90,1\% (91/116) de los controles (Especificidad) (Tabla 3).

\section{Modelo de estilos de vida (modelo 2)}

En el segundo modelo quedaron las variables: primaria incompleta, primaria completa y secundaria incompleta. El riesgo de tener un recién nacido de BPN, fue 5,10 veces en las mujeres con primaria incompleta, 2,76 veces en primaria completa y 2,52 veces en secundaria incompleta, en los 3 casos comparados con mujeres que tenían formación profesional. El segundo modelo clasificó correctamente al 62,5\% (140/233) de los individuos; $64,3 \%(72 / 117)$ de los casos y 60,7\% (68/116) de los controles (Tabla 3).

Tabla 3. Análisis multivariado modelos 1, 2, 3 y 4

\begin{tabular}{|c|c|c|c|c|c|}
\hline \multirow{2}{*}{ Variables } & \multirow{2}{*}{$\begin{array}{l}\text { Modelo } 1 \\
\text { (Biología } \\
\text { humana) }\end{array}$} & \multirow{2}{*}{$\begin{array}{l}\text { Modelo } 2 \\
\text { (Estilos de } \\
\text { vida) }\end{array}$} & \multirow{2}{*}{$\begin{array}{c}\text { Modelo 3 } \\
\text { (Servicios de } \\
\text { salud) }\end{array}$} & \multicolumn{2}{|c|}{ Intervalo de Confianza 90\% } \\
\hline & & & & Superior & Inferior \\
\hline Edad joven (10 - 19 años) & $4,24^{*}$ & & & 1,73 & 10,39 \\
\hline Índice de Masa Corporal (IMC) & $0,93^{*}$ & & & 0,88 & 0,98 \\
\hline Infección vaginal al final del embarazo & $2,95^{*}$ & & & 1,19 & 7,31 \\
\hline Edad gestacional a termino & $0,33^{*}$ & & & 0,25 & 0,43 \\
\hline Primaria incompleta & & $5,10^{*}$ & & 1,62 & 16,02 \\
\hline Primaria completa & & $2,76^{*}$ & & 1,49 & 5,12 \\
\hline Secundaria incompleta & & $2,52^{*}$ & & 1,47 & 4,31 \\
\hline Afiliación SGSS (Subsidiado) & & & $2,12^{*}$ & 1,16 & 3,87 \\
\hline Remitida de otro lugar & & & $2,78^{*}$ & 1,68 & 4,59 \\
\hline Hospitalización en el embarazo & & & $2,89 *$ & 1,75 & 4,77 \\
\hline Número de controles prenatales (5 o más) & & & $0,89^{*}$ & 0,81 & 0,98 \\
\hline VPC & $84,8 \%$ & $62,5 \%$ & $63,9 \%$ & & \\
\hline
\end{tabular}

VPC: Porcentaje de correcta clasificación

*Variables que fueron estadísticamente significativas

\section{Modelo de servicios de salud (modelo 3)}

En el tercer modelo quedaron las variables afiliación al Sistema General de Seguridad Social (SGSS), en el régimen subsidiado, remitida de otro lugar, hospitalización en el embarazo, más de cinco controles prenatales. El riesgo de tener un recién nacido de BPN fue 2,12 veces en las mujeres que se encontraban afiliadas al régimen subsidiado comparado con quienes estaban afiliadas al régimen contributivo, 2,78 veces en las mujeres que fueron remitidas desde otros niveles de atención comparado con los que llegaron directamente al hospital, 2,89 veces en las mujeres que se hospitalizaron durante el embarazo comparado con las que no. Por cada aumento en un CPN, el riesgo de tener un recién nacido de BPN presentó una reducción promedio de $11 \%$. El tercer modelo clasificó correctamente al 63,9\% (140/233) de los individuos; 65,7\%; (71/117) de los casos y 63,9\% (69/116) de los controles (Tabla 3).

\section{Análisis multivariado - Modelo resumen}

Se corrió un modelo resumen que integró las variables estadísticamente significativas de los tres modelos anteriores (biología humana, estilos de vida y organización sanitaria), como resultado se obtuvo 
que el riesgo de tener un hijo con bajo peso al nacer fue 7,79 veces en mujeres con edades entre los 10-19 años, 4,96 veces en quienes presentaban infección vaginal durante el embarazo, 10,93 veces en mujeres con primaria incompleta, 2,9 veces en aquellas con primaria completa, 4,96 veces en quienes estaban afiliadas al régimen subsidiado. El riesgo fue menor a medida que aumentaba el IMC y la edad gestacional, por cada incremento en una unidad de IMC el riesgo de tener un recién nacido de bajo peso se redujo en un $10 \%$, y por cada incremento en una semana de gestación el riesgo disminuyó en 76\% (Tabla 4).

Tabla 4. Análisis multivariado modelo integrado

\begin{tabular}{lrrr}
\hline \multirow{2}{*}{ Variables explicativas } & \multirow{2}{*}{$\begin{array}{c}\text { Odds } \\
\text { Ratio }\end{array}$} & \multicolumn{2}{c}{ IC 90\% } \\
\cline { 3 - 4 } & (OR) & Inferior & Superior \\
\hline Edad joven (10 - 19 años) & 7,79 & 2,61 & 23,23 \\
IMC & 0,90 & 0,84 & 0,96 \\
Infección vaginal al final del & 4,0 & 1,26 & 12,66 \\
embarazo & & & \\
Edad gestacional a término & 0,23 & 0,16 & 0,34 \\
Primaria incompleta & 10,93 & 1,51 & 79,13 \\
Primaria completa & 2,94 & 1,06 & 8,13 \\
Afiliación SGSS (subsidiado) & 4,96 & 1,71 & 14,41 \\
\hline
\end{tabular}

\section{Discusión}

El modelo logístico resumen identificó al embarazo en niñas y adolescentes (10-19 años) como factor de riesgo para el $\mathrm{BPN}(O R=7,79, I C 90 \%=2,61-23,23)$, lo cual coincide con estudios previos, uno de ellos realizados en Etiopía que demostró que las mujeres con edades menores a 20 años presentaban 3 veces más riesgo de $\mathrm{BPN}(O R=3,0, I C 95 \%=1,65-5,73)^{(14)}$.

El incremento en el IMC y en las semanas de gestación, resultaron ser factores protectores, en una revisión sistemática realizada en el 2018, los investigadores concluyeron que el IMC está positiva y significativamente relacionado con el peso al nacer, encontrando que las madres que comienzan la gestación con bajo peso, tienen un mayor riesgo de concebir niños con BPN, al igual que las mujeres obesas y con sobrepeso tienen un mayor riesgo de tener hijos macrosómicos(15).

Variables biológicas con serología reactiva al inicio y al final de embarazo y antecedente de infección de vías urinarias no se relacionaron con BPN, hallazgo que pudo ser afectado por el subregistro de datos en el CLAP, ficha que era valorada en el estudio, la mayoría de las maternas eran remitidas y no se contaba con la historia clínica materna completa.

La infección vaginal por su parte, resultó ser un factor de riesgo para BPN, hallazgo reportado por otros investigadores, como el estudio realizado en Santiago de Cuba, el cual mostró que una de las enfermedades más frecuentes durante la gestación y que incidió mayormente en el BPN en el grupo de casos fue la infección vaginal con $62,5 \%$ de ocurrencia(6).

Aunque en la presente investigación se identificó una prevalencia alta de infección vaginal (19\%), no a todas las madres se les realiza tamizaje para estreptococo del grupo B y aunque la Guía de Práctica Clínica para la Detección Temprana de Alteraciones del Embarazo de MSPS(16) establece que toda muestra debe ser enviada al laboratorio de control, el personal de salud que realiza los controles prenatales, no ordena los laboratorios completos, y si es remitida la gestante en muchas oportunidades no se le toma la muestra vaginal, y algunas gestantes no regresan por el resultado. El estudio de Etiopía también menciona que los problemas de salud durante el embarazo generan alto riesgo de BPN $(O R=6,3, I C 95 \%=2,75$ $14,48)^{(14) .}$

En cuanto a las variables del estilo de vida, el nivel educativo resultó estadísticamente significativa en el análisis multivariado y solo el nivel primaria quedó en el modelo integrado, siendo mayor la fuerza de asociación cuando este nivel no se culmina (primaria incompleta), la educación se relaciona con muchas condiciones importantes asociadas al embarazo, como el acceso a métodos de planificación familiar y asistencia a controles prenatales(17). Un estudio realizado en hospitales del Ministerio de Salud de Perú, reportó que el nivel educativo analfabeta/primaria se relaciona con BPN $(O R=1,4$, $I C 95 \%=1,31-1,50)(18)$. Por su parte, una investigación realizada en población italiana reportó que tener educación superior fue un factor protector contra la ocurrencia del BPN $\left(O R=0,78\right.$; IC 95\% 0,70-0,81) ${ }^{(19)}$.

La única variable relacionada con los servicios de salud que quedó incluida en el modelo resumen, fue la afiliación al régimen subsidiado, identificándose como factor de riesgo. En Colombia la afiliación a este régimen predomina en poblaciones con bajas condiciones socioeconómicas, lo cual, a su vez, puede favorecer la suma de múltiples factores como ingresos económicos bajos, estilos de vida 
inadecuados, pocos cuidados prenatales, deficiente situación laboral y cuidados médicos en general, entre otros(20). Un estudio que analizó datos de cuatro cohortes de gestantes, en Brasil, encontró que en todas las cohortes de nacimientos, las madres más pobres tenían mayores probabilidades de tener neonatos con bajo peso al nacer o pequeños para la edad gestacional(21). En Colombia, entre 2002 a 2011, se encontró que las mujeres con mayor probabilidad de tener un recién nacido con BPN, fueron las pertenecientes al régimen subsidiado $(O R=1,10$, IC95\%=1,07-1,09)(3).

Una variable que no fue incluida en el modelo resumen, pero que es muy importante analizar fue la asistencia al control prenatal. Esta variable resultó estadísticamente significativa en el modelo de regresión lineal de los servicios de salud (modelo 3). Se identificó que a mayor asistencia al CPN, menor riesgo de BPN; según la Guía de Práctica Clínica(16) los CPN deben iniciar en el primer trimestre del embarazo, por lo tanto, entre mayor sea el contacto con los servicios de salud, de manera oportuna, más rápido se podrá diagnosticar y tratar enfermedades que pongan en peligro la vida de la madre y del neonato.

Un estudio realizado con 751 adolescentes atendidos en un Hospital Público de Rio de Janeiro encontró que entre los determinantes asociados a BPN, está tener menos de 6 consultas prenatales $(O R=4,29$, $I C 95 \%=1,55-11,83)^{(22)}$. Un estudio realizado en Colombia, encontró una asociación entre la inasistencia a control prenatal y mayor probabilidad de BPN $\left(O R=1,90, I_{C} 95 \%=1,88-1,97\right)^{(3)}$.

Esta investigación permitió identificar los principales factores relacionados con el BPN en la población atendida en el HUDN; sin embargo, es importante considerar algunas limitaciones del mismo; por ejemplo, el sesgo de memoria, común en los estudios de casos y controles el cual genera la posibilidad de que los controles recuerden menos los eventos pasados, comparados con los casos, por lo cual además de la encuesta la información relacionada con la atención en salud fue verificada en la historia, aunque variables como los antecedentes familiares pueden continuar viéndose afectadas.

Por otra parte, tal como lo declara el sesgo de Berkson(23), es posible que las mujeres que tienen partos institucionales pueden no ser representativas de la población general, teniendo en cuenta que en el departamento de Nariño existe un alto porcentaje de población indígena, y rural cuyos partos suelen ser atendidos por parteras. Es importante considerar que la mayoría de las mujeres pertenecían a estratos bajos, lo cual representa a la población que en general es atendida en el HUDN, pero no quiere decir que represente el mayor porcentaje de estrato socioeconómico de los habitantes de la ciudad de Pasto, por lo tanto, es difícil aplicar los hallazgos de este estudio a poblaciones diferentes a las estudiadas.

Si bien existen múltiples estudios que ya han explorado los factores de riesgo asociados al BPN, entre los que se encuentran: edad materna, multiparidad, bajo nivel educativo, pobreza, mujeres solteras, inadecuado control prenatal(3-5,14,18-22), infecciones del tracto genitourinario, anemia, preeclampsia, ruptura prematura de membranas(6), malnutrición materna, entre otros determinantes sociales y ambientales de la salud $(7,15)$, las autoridades sanitarias del nivel municipal, departamental y nacional deberían insistir en la planeación de los servicios ofertados, a partir de un enfoque diferenciado, basado en los riesgos detectados en la población local, debido a que existen diferencias importantes entre los territorios sociales.

Las políticas públicas de alguna forma han tenido desaciertos ya que van dirigidas a todo un país, sin tener en cuenta las particularidades locales y el efecto de las características sociales, culturales y demográficas, y que la prevalencia de los factores de riesgo está relacionada con las mismas, por tanto, actúan de manera diferente entre regiones.

\section{Conclusiones}

Los factores de riesgo identificados en este estudio a través del modelo final fueron, madres con edades entre los 10-19 años, presencia de infección vaginal durante el embarazo, contar con primaria incompleta o con primaria completa como nivel educativo, afiliación al régimen subsidiado, los cuales se pueden detectar y tratar precozmente para impedir, acortar o paliar su evolución y consecuencias con enfoque de salud pública.

Entre los factores protectores identificados, se encuentran el incremento en el IMC y las semanas de gestación 


\section{Recomendaciones}

Teniendo en cuenta que una de las variables relacionada de manera importante con el BPN en la población estudiada, fue el grado de escolaridad de la gestante, algunas de las recomendaciones más relevantes que surgen a partir de la presente investigación va encaminada a la necesidad de continuar mejorando la educación sanitaria de las mujeres en edad reproductiva sobre todo en edad escolar, con metodologías pedagógicas que les permitan ampliar sus conocimientos sobre métodos de planificación familiar, además de fortalecimiento del proyecto de vida de las jóvenes. Desde la perspectiva del grupo investigador, la educación debe ser gratuita y accesible a todo tipo de población.

Promover en las mujeres gestantes, el compromiso de asistir oportunamente a los controles médicos necesarios.

Es importante integrar los resultados de esta investigación a los planes territoriales, a la programación de las instituciones prestadoras de servicios de salud (IPS) y a las estrategias actuales de promoción de la salud de la región.

Garantizar que en las IPS se brinde una atención integral personalizada las gestantes, realizando seguimiento y monitoreo de los riesgos para la salud, para que el embarazo llegue a buen término y se asegure las mejores condiciones posibles al recién nacido.

\section{Agradecimientos}

A las pacientes y al Hospital Universitario Departamental de Nariño, dado que su participación hizo posible la investigación.

Conflicto de intereses: Los autores no declaran ningún conflicto de intereses.

\section{Referencias}

1. Organización Mundial de la Salud (OMS). Metas mundiales de nutrición 2025. Documento normativo sobre bajo peso al nacer [Internet]. 2017. Disponible en: https://apps.who.int/iris/bitstream/handle/10665/25573 3/WHO_NMH_NHD_14.5_spa.pdf

2. Vilanova CS, Hirakata VN, de Souza Buriol VC, Nunes M, Zubaran Goldani M, Homrich da Silva C. The relationship between the different low birth weight strata of newborns with infant mortality and the influence of the main health determinants in the extreme south of Brazil. Popul Health Metr [Internet]. 2019;17(1):15. DOI: 10.1186/s12963-0190195-7.
3. Estrada-Restrepo A, Restrepo-Mesa SL, Ceballos Feria N del C, Mardones Santander F. Factores maternos relacionados con el peso al nacer de recién nacidos a término, Colombia, 2002-2011. Cad Saúde Pública [Internet]. 2016;32(11):e00133215. Disponible en: http://www.scielo.br/scielo.php?script=sci_abstract\&pid=S 0102-311X2016001105006\&lng=en\&nrm=iso\&tlng=es

4. Magalhães Moreira AI, Moreira de Sousa PR, Sarno F. Low birth weight and its associated factors. Einstein São Paulo [Internet]. 2018;16(4):eA04251. Disponible en: http://www.scielo.br/scielo.php?script=sci_abstract\&pid=S 1679-45082018000400202\&lng=en\&nrm=iso\&tlng=en

5. Mahecha-Reyes E, Grillo-Ardila CF. Maternal Factors Associated with Low Birth Weight in Term Neonates: A Casecontrolled Study. Rev Bras Ginecol Obstet [Internet]. 2018;40(8):444-9. Disponible en: https://pubmed.ncbi.nlm.nih.gov/30142664/

6. Bertrán Bahades J, Muguercia Silva JL, Verdaguer Pérez L, Morejón Rebelo I, García Kindelán M de la C. Factores de riesgo asociados al bajo peso al nacer en un área de salud de Santiago de Cuba. MEDISAN. 2019;23(4):619-31. Disponible en:

http://www.medisan.sld.cu/index.php/san/article/view/1 968

7. Borah M, Agarwalla R. Maternal and socio-demographic determinants of low birth weight (LBW): A communitybased study in a rural block of Assam. J Postgrad Med [Internet]. 2016;62(3):178-81. Disponible en: https://pubmed.ncbi.nlm.nih.gov/27320953/

8. Ministerio de salud y protección social de Colombia. Actualización de los Lineamientos Técnicos para la implementación de Programas Madre Canguro en Colombia, con énfasis en la nutrición del neonato prematuro o de bajo peso al nacer [Internet]. 2017. Disponible en: https://www.minsalud.gov.co/sites/rid/Lists/BibliotecaDi gital/RIDE/DE/implementacion-programa-canguro.pdf

9. Observatorio así vamos en salud. Prevalencia De Bajo Peso al Nacer - Georeferenciado [Internet]. www.asivamosensalud.org. 2021. Disponible en: https://www.asivamosensalud.org/indicadores/seguridadalimentaria-y-nutricional/prevalencia-de-bajo-peso-alnacer

10. Ministerio de salud de Colombia. Resolución 8430 de 1993 [Internet]. 1993.1 Disponible https://www.minsalud.gov.co/sites/rid/Lists/BibliotecaDi gital/RIDE/DE/DIJ/RESOLUCION-8430-DE-1993.PDF

11. Schlesselman J. Case-Control Studies: Design, Conduct, Analysis. New York: Oxford University Press; 1982. Disponible

en: https://books.google.com.co/books?id=50kyFkYOn0QC\&pr intsec $=$ frontcover $\& \mathrm{hl}=\mathrm{es} \# \mathrm{v}=$ onepage $\& \mathrm{q} \& \mathrm{f}=$ false

12. Rothman K. Epidemiología moderna. Ediciones Díaz de Santos. Vol. 1. Madrid, España; 1987. 398 p. Disponible en: https://www.editdiazdesantos.com/libros/rothmankenneth-j-epidemiologia-moderna-L01000680401.html

13. Lalonde M, Government of Canada. A New Perspective on the Health of Canadians. A working document [Internet]. Ottawa; 1974. Disponible en: http://www.phac-aspc.gc.ca/phsp/pdf/perspect-eng.pdf

14. Demelash H, Motbainor A, Nigatu D, Gashaw K, Melese A. Risk factors for low birth weight in Bale zone hospitals, SouthEast Ethiopia: a case-control study. BMC Pregnancy Childbirth [Internet]. 2015;15:264. Disponible en: https://pubmed.ncbi.nlm.nih.gov/26463177/p 
15. Megías Patón C, Prados-Ruiz JL, Rodríguez-Blanque R, Sánchez-García JC. El IMC durante el embarazo y su relación con el peso del recién nacido. J Negat No Posit Results [Internet]. 2018;3(3):215-24. Disponible en: https://revistas.proeditio.com/jonnpr/article/view/2173

16. Ministerio de Salud y Protección Social de Colombia. Guías de Práctica Clínica para la prevención, detección temprana y tratamiento de las complicaciones del embarazo, parto o puerperio [Internet]. 2013. Disponible en: https://www.minsalud.gov.co/sites/rid/Lists/BibliotecaDi gital/RIDE/INEC/IETS/Gu\%C3\%ADa.completa.Embarazo.P arto.2013.pdf

17. Ministerio de Salud y Protección Social, Equipo Sexualidad y Derechos Sexuales y Reproductivos. Política Nacional de Sexualidad, Derechos Sexuales y Derechos Reproductivos [Internet]. 2017. Disponible en: https://www.minsalud.gov.co/sites/rid/Lists/BibliotecaDi gital/RIDE/DE/LIBRO\%20POLITICA\%20SEXUAL\%20SEPT \%2010.pdf

18. Ticona Rendón M, Huanco Apaza D, Ticona Vildoso M. Incidencia y factores de riesgo de bajo peso al nacer en poblacion atendida en hospitales del Ministerio de Salud del Perú. Ginecol Obstet México [Internet]. 2012;80(2):51-60. Disponible https://www.medigraphic.com/pdfs/ginobsmex/gom2012/gom122b.pdf
19. Cantarutti A, Franchi M, Monzio Compagnoni M, Merlino L, Corrao G. Mother's education and the risk of several neonatal outcomes: an evidence from an Italian population-based study. BMC Pregnancy Childbirth [Internet]. 2017;17(1):221. Disponible en: https://www.ncbi.nlm.nih.gov/pmc/articles/PMC5508478

20. Departamento Nacional de Planeación de Colombia - DNP. Aseguramiento. [actualizado 2016 Nov 11]. Disponible en: https://www.dnp.gov.co/programas/desarrollosocial/subdireccion-de-salud/Paginas/aseguramiento.aspx

21. Sadovsky ADI, Matijasevich A, Santos IS, Barros FC, Miranda AE, Silveira MF. LBW and IUGR temporal trend in 4 population-based birth cohorts: the role of economic inequality. BMC Pediatr [Internet]. 2016;16:115. Disponible en: https://pubmed.ncbi.nlm.nih.gov/27473678/

22. Pinto Belfort G, de Souza Santos MMA, da Silva Pessoa L, Rebelo Dias J, Petronilho Heidelmann S, Saunders C, et al. Determinants of low birth weight in the children of adolescent mothers: a hierarchical analysis. Ciênc \& Saúde Coletiva [Internet]. 2018;23(8):2609-20. Disponible en: http://www.scielo.br/scielo.php?script=sci_abstract\&pid=S 1413-81232018000802609\&lng=en\&nrm=iso\&tlng=en

23. Argimon Pallás JM, Jiménez Villa J. Métodos de investigación clínica y Epidemiológica. 4.a ed. España: Elsevier; 2013. Disponible en: https://www.elsevier.com/books/metodosde-investigacion-clinica-y-epidemiologica/9788481747096 